\title{
Bile acid CoA ligase deficiency and defective amidation
}

INSERM

\section{Source}

INSERM. (1999). Orphanet: an online rare disease and orphan drug data base. Bile acid COA ligase deficiency and defective amidation. ORPHA:276066

Bile acid CoA ligase deficiency and defective amidation is an anomaly of bile acid synthesis (see this term) characterized by fat malabsorption, neonatal cholestasis and growth failure. 